\title{
Compulsory Islanding Transition Strategy Based on Fuzzy Logic Control for a Renewable Microgrid System
}

\author{
Jiexing Wan $(\mathbb{D}$, Wei Hua, and Baoan Wang \\ School of Electrical Engineering, Southeast University, Nanjing 210096, China \\ Correspondence should be addressed to Jiexing Wan; 230179195@seu.edu.cn
}

Received 3 March 2021; Accepted 5 May 2021; Published 18 May 2021

Academic Editor: Xiao-Shun Zhang

Copyright ( $\odot 2021$ Jiexing Wan et al. This is an open access article distributed under the Creative Commons Attribution License, which permits unrestricted use, distribution, and reproduction in any medium, provided the original work is properly cited.

\begin{abstract}
Advantages of microgrid integrated with a renewable energy system have been acknowledged in more and more applications. Operating steadily during different modes is of great significance, and different modes transferring must be transient, seamless, and reliable to maintain the continuous electricity supply for loads, especially sensitive ones. As a semicontrolled switch that is unable to shut instantly until the zero point of its current, thyristors should be forced to be turned off by certain strategies to minimise the influence brought by the delay of modes transferring. In this paper, a compulsory turn-off strategy based on fuzzy logic control of converter output voltage is proposed. With the strategy, an alterable reverse voltage is applied across the conducting thyristor by dynamically adjusting the output voltage reference instruction and closed-loop control parameters depending on the changing rate rather than detecting the polarity of voltage or current. Constraints from acquisition of analog data and error of digital calculation will be eliminated. Finally, simulation and experimental results verify the effectiveness of the proposed strategy.
\end{abstract}

\section{Introduction}

In recent years, motivated by the climate change and unsustainable consumption on fossil fuels for electricity generation, establishment of the microgrid (MG) system with renewable energy conversion such as distributed generating (DG) or battery storing (BS) has steadily increased [1-3]. The MG system is capable of supplying uninterrupted power for critical loads independently with the flexibility of multiple working modes under planned outage or unintentional faults such as short circuit, disturbance, and blackout [4-7]. Because of different control objectives, the mode transition of the MG system, especially when it disconnects from the utility, must be transient, seamless, and reliable so that influence caused by the intermediate process can be mitigated or avoided even better without any current surge or voltage fluctuation [8].

Studies have been carried out on smooth transferring of output. In [9], a master inverter is set to build up the AC voltage and other inverters can still work in the grid-tied mode to supply continuous power. Unified schemes based on indirect current control are devised to impose no alteration to the control structure so that the output voltage and injected current can transmit smoothly with no shock [10-12]. In [13, 14], smooth transition mechanisms based on state observer are discussed to maintain the system variables within acceptable limits. In [15-18], a unified scheme based on droop control is designed to only change the voltage reference rather than control structure so that inrush currents and voltage distortions of mode transfer can be eliminated. In [19-22], fuzzy-based parameter regulation is implemented to improve the performance of dynamic response and stability when transferring.

However, before getting power supply from the MG system, loads should disconnect from the utility completely $[23,24]$. Inadequate attention has been applied on switchover transients of the connecting devices between the utility and the MG system, which leaves several challenges to be further researched. Generally, it takes around $1 \mathrm{~s}$ (second) for the relays to switch off completely and around $0.5 \mathrm{~s}$ for mechanical breakers [25] while power electronic switches have much faster on-off response [26]. In spite of this, the MG system is usually connected to the utility through switches consisting of thyristors rather than IGBTs, GTOs 
etc., in AC bus from current-carrying capability and the practicability point of view, especially in high voltage and current occasions $[27,28]$. In [29-31], it takes from $1 / 4$ to $1 / 2$ line cycle for the MG system to disconnect from the utility at zero crossing of the grid current and 5 line cycle to ensure the whole procedure at most, which is far beyond the requirement of power continuity for sensitive loads [32]. Even worse, power quality of the MG system may be further deteriorated by the turn-off delay. So, it is of great significance to take measures to ensure the fast and smooth switching of thyristors.

Compensation strategies are introduced in $[33,34]$ that the injecting current of the inverter is regulated to follow the instantaneous value of load when the off-grid transfer signal starts up; then, the current of thyristors can be reduced to zero and enter the blocking state quickly. But, the deficiencies are also obvious. For one thing, the injecting current of the inverter is difficult to be controlled exactly as the load current, especially when the load is nonlinear or light. On the contrary, if the load is heavy, the voltage will undergo a distortion because of the current surge. An LC series resonance circuit is designed to reverse the deviation of voltage on thyristors to accelerate the current decaying [35] while it can be implemented simply in the case of voltage sag rather than any other anomalies and the resonance is susceptible to variation of characteristics. In [36], two control methods for the transition by means of inverter output voltage amplitude and phase regulation, which depend heavily on the accurate polarity of the static transfer switch current, and measures dealing with inaccurate judgment are not explained. When the instantaneous amplitude of conducting current is too small to be sampled by the sensors (e.g., near zero) of the system controller, the polarities will not be recognized accurately. Then, there will be a high probability that the MG system fails to disconnect from the utility before working as a voltage source. To solve this problem, the MG system controller can equip a highprecision and wide-range sampling circuit in its detecting section, which may take much more cost. On the other hand, it can also set a minimum threshold to escape transition when the current or voltage is beyond the linear sampling region, which may jeopardize the power continuity and stability of local loads.

Taking the limitations of research mentioned above into consideration, this paper proposes a new strategy based on fuzzy logic control of converter output voltage to force to turn the MG thyristors off without detecting the voltage or the current polarity at the moment of islanding so that the transitory time of utility disconnecting can be minimized effectively. Firstly, an initial voltage conference is set for the MG converter to form a voltage deviation between the anode and cathode of the conducting thyristor. Then, the voltage conference is regulated according to fuzzy logic rules based on the following variation trends of its current until the conducting thyristor is turned off. The proposed method makes sure that the MG system can accomplish islanding transition rapidly at any time and any circumstance and can ensure the reliability for MG customers more comprehensively during the occurrence of utility anomaly.
The main contributions of this paper are listed as follows:

(1) The mathematical model of compulsory turning off for thyristors in the MG system is demonstrated

(2) In contrast to the existing control methods, an islanding transition strategy without detecting the polarity is introduced on the basis of the mathematical relationship between the time of compulsory turnoff with the parameters of main circuit and control system

(3) Fuzzy rules of the compulsory disconnecting strategy for the thyristors through the output voltage of the MG converter are proposed to ensure islanding transition rapidly at any time and any circumstance

The remainder of this paper is organized as follows. Four different operational modes of the MG system are presented in Section 2 with a brief review about the necessity of control scheme studied in this paper. Section 3 demonstrates the control principle of compulsory turnoff for the thyristors in the MG system by the equipped converter and formulates the mathematical relationship between turn-off time with the main circuit and control parameters on the basis of a single-phase schematic diagram of the MG system. The strategy of compulsory islanding based on fuzzy logic to regulate the output voltage of the converter in the MG system is presented in Section 4. Simulation with four voltage and current situations and, practically, experimental results are displayed in Section 5 to prove the validity and effectiveness of the proposed method. Finally, conclusions are duly down.

\section{System Description}

2.1. Structure and Working Modes of the MG System. A typical single-phase structure diagram and controlling block of the MG power supply system integrated with the utility is mapped in Figure 1. In detail, local loads and the MG system are connected in parallel to the utility by thyristors. A solar cell and energy storage battery are connected to the DC bus and provide energy support for different operational modes of the MG system. The converter is connected to the utility through the LC filter. The MG power controller monitors the voltage of the public grid as well as the DC bus in real time and outputs driving signals to turn on/off the thyristors in order to change the connecting state between the MG system and the utility. Space vector pulse width modulation (SVPWM) is used to reduce the switching harmonics and to improve the utilization of DC voltage.

Two operational modes of the MG system are further depicted in Figure 1, which are utility-connected mode and stand-alone mode. The MG power controller changes the strategy with a software switch for the converter to achieve different output targets by working as a controlled current or voltage source relatively according to the ON/OFF state of thyristors. However, the utility-connected and stand-alone mode, which can be also defined as steady-state modes, should not transfer from one to another directly in order to avoid saturation or mutation of controlling. As is shown in 


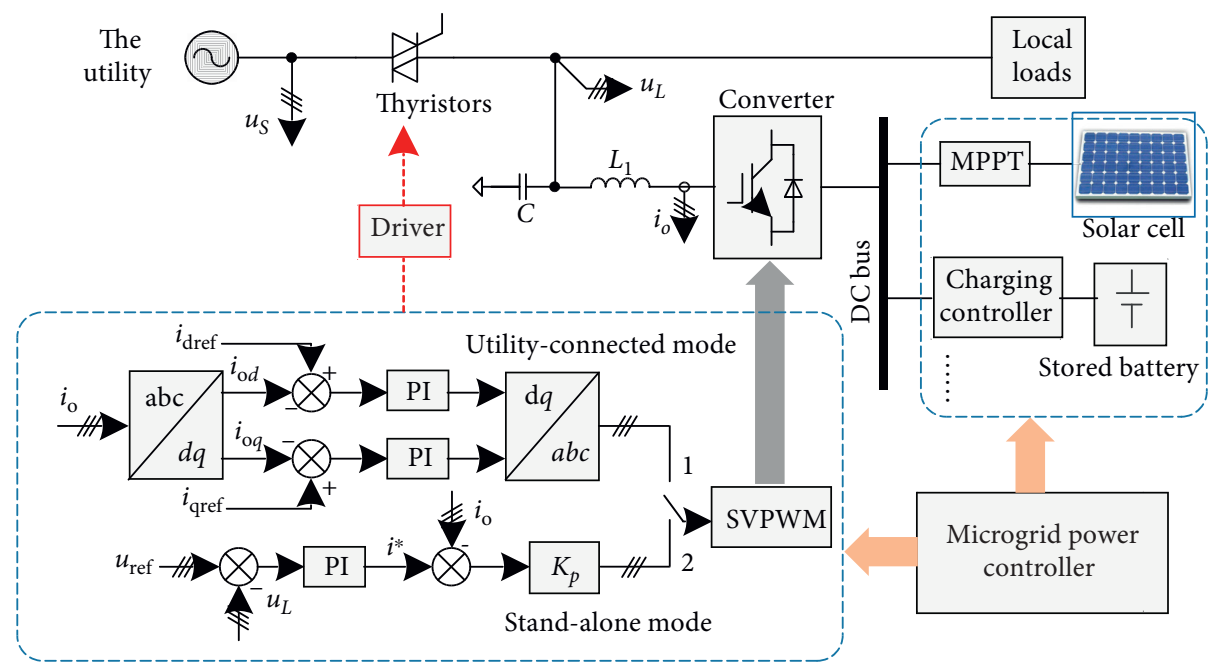

Figure 1: Typical single-phase structure diagram and control methods of the MG system.

Figure 2, between the utility-connected mode (mode 1) and the stand-alone mode (mode 2), there are other two transient-state modes for smooth transferring to help to prevent any sudden voltage or current changes to the load as well as to the grid, which are presynchronization (mode 3 ) and islanding transition (mode 4).

2.1.1. Mode 1: Utility-Connected Mode. If the utility is stiff, thyristors are turned $\mathrm{ON}$ and the MG system works at the utility-connected mode. Local loads get voltage supply from the utility. The MG converter works as a current source, which can both maintain the inactive power balance and provide active power to reduce electricity cost from the main grid if necessary. In addition, the stored battery of the distributed generating part can get charged during this mode.

2.1.2. Mode 2: Stand-Alone Mode. When an abnormal grid condition occurs, the thyristors are turned OFF and the MG system disconnects from the utility. In this situation, the MG system is working at the stand-alone mode and local loads get power from the MG system independently. The MG converter transfers its control strategy from current control to voltage control to ensure the continuous voltage supply for the operation of local loads.

2.1.3. Mode 3: Presynchronization. When the MG system is transferring from the stand-alone mode to utility-connected mode, the thyristors are switching from the OFF state to ON state and the converter adjusts the referencing instruction in accordance with the voltage of the utility [7]. If the deviation between the MG converter output and the utility voltage reduces to an acceptable range, the thyristors will be turned on and the converter will get reconnected to the utility along with local loads. This process is presynchronization [13, 33-37]. Presynchronization is the operational process that the converter regulates its output voltage (including amplitude, frequency, and phase) constantly as a controlled voltage source after the utility is restored normally, which is totally controllable and predictable.

2.1.4. Mode 4: Islanding Transition. In contrast, the transition that is from utility-connected to stand-alone mode brings much more uncertainties due to the switching state of thyristors from the ON state to OFF state. When there is scheduled maintenance or planned islanding, the MG power controller can schedule the transferring of working modes in advance. If it is unplanned, which might be occasional voltage sag, swell, interruption, or even worse, then the MG system must respond in time to ensure the consecutiveness of power supply. Firstly, the thyristors should be shut off so that local loads can disconnect from the utility, and then, the MG controller should change the strategy to transfer its operation to the stand-alone mode.

However, for the MG system with thyristors as $\mathrm{AC}$ switches, the turn-off time ranges from microseconds to half of the fundamental cycle, which is far beyond the acceptance of sensitive loads. Therefore, it is of great significance to realize the fast switching of the working mode at any moment and to reduce the impact of the switching interval.

2.2. Compulsory Turnoff of Thyristors in the MG System. As a kind of semicontrolled power electronic device, once the thyristor begins to conduct, the gate loses control and the switch-on process continues until the anode-cathode current reduces to zero or below the holding value [36]. This zero-crossing turn-off prototype can be defined as natural turnoff while measures that applied to accelerate the decay of $I_{\mathrm{AK}}$ can be defined as compulsory turnoff.

Figure 3 shows the equivalent single-phase schematic of the integrated power supply system. The positive direction of the current in each branch is determined as the arrows in the figure. The voltage of the utility is represented as $u_{s}$ while the equivalent line impedance of the utility is $Z_{s}$. The symbol $i_{s}$ is the current crossing thyristors. The load of the MG system is $Z_{L}$, while the voltage and current are $u_{L}$ and $i_{L}$. As for the DG 


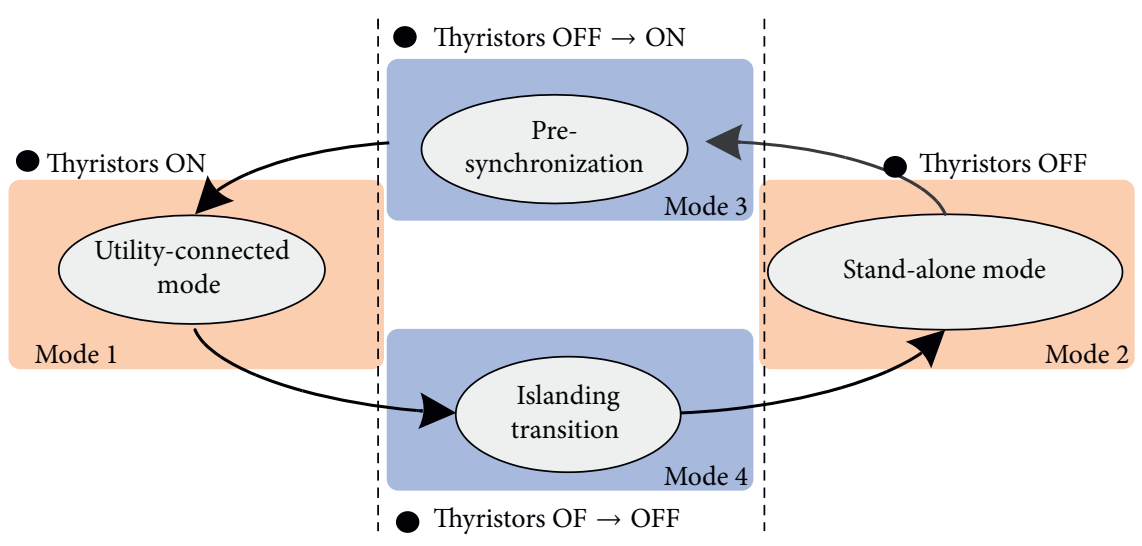

Steady-state modes

Transient-state modes

FIGURE 2: Converting relationship of different operational modes in the MG system.

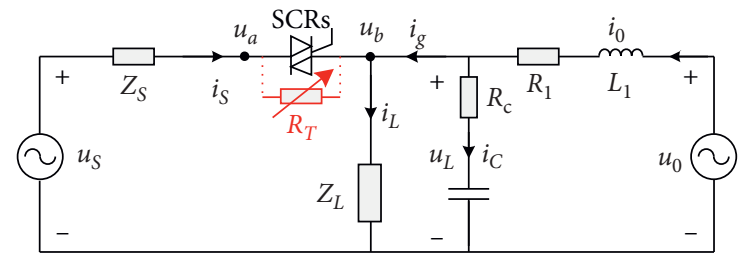

FIGURE 3: Equivalent single-phase schematic of the MG system.

system, $u_{0}$ and $i_{0}$ are the output voltage and current of the converter, respectively, while $R_{1}$ and $R_{c}$ are the parasitic resistances of the LC filter.

When the converter works as a voltage source, the amplitude and phase of the output voltage $u_{0}$ are adjustable through the MG controller. If the voltage output of converter makes the anode-cathode voltage $U_{\mathrm{AK}}$ positive, the thyristor will continue to conduct until the next zero point of current. Even worse, an inrush and uncontrolled current caused by the parallel connecting of two voltage sources will trigger the protection mechanism [7]. If the MG converter makes the voltage $U_{\mathrm{AK}}$ negative, the thyristor can be turned off in a very short time and adverse effects of modes transferring on local loads, especially sensitive loads, can be eliminated [37]. Ignoring the voltage drop of thyristors, there are four situations according to the instantaneous value of the current and the voltage to satisfy the condition of compulsory turnoff:

(1) If $i_{s}>0$ and $u_{a}>0$, then $u_{b}>u_{a}>0$

(2) If $i_{s}>0$ and $u_{a}<0$, then $u_{b}>u_{a}$

(3) If $i_{s}<0$ and $u_{a}>0$, then $u_{b}<u_{a}$

(4) If $i_{s}<0$ and $u_{a}<0$, then $u_{b}<u_{a}<0$

This can be summarized as

$$
\begin{cases}u_{b}-u_{a}>0, & i_{s}>0, \\ u_{b}-u_{a}<0, & i_{s}<0 .\end{cases}
$$

During compulsory turnoff, the output voltage reference $u_{\text {ref }}$ of the MG converter can be expressed as

$$
u_{\text {ref }}=u_{a}+\Delta u
$$

where $\Delta u$ is a kind of regulative variable that can form a voltage deviation between the utility and the converter and $u_{a}$ is the measuring point of system voltage.

When the conducting thyristor begins to turn off, it can be seen as a variable resistor $R_{T}$ equivalently [28], of which the relationship between resistance and current under different voltage is shown in Figure 4. $I_{\mathrm{AK}}$ represents the current flowing from the anode to cathode of the conducting thyristor while $U_{\mathrm{KA}}$ represents the reverse voltage. It is indicated that the resistance $R_{T}$ increases as $I_{\mathrm{AK}}$ decreases. In addition, if the resistance keeps its value in $R_{1}$, a greater $U_{\mathrm{KA}}$ will bring a faster decaying speed for $I_{\mathrm{AK}}$. Meanwhile, if the current keeps its value in $I_{2}$, the resistance will increase along with the $U_{\mathrm{KA}}$, which means a greater suppression for $I_{\mathrm{AK}}$.

The relationship between the output voltage and the decaying time of current can be calculated during the compulsory turn-off process. For the studied system in Figure 3, there are equations as follows:

$$
\left\{\begin{array}{l}
u_{L}=u_{0}-R_{1} i_{0}-L_{1} \frac{\mathrm{d} i_{0}}{\mathrm{~d} t}, \\
i_{L}=\frac{u_{L}}{Z_{L}}=i_{S}+i_{o}-i_{C}
\end{array}\right.
$$

The parasitic resistances $R_{1}$ and $R_{C}$ are omitted in order to facilitate the analysis; then, the current $i_{C}$ can be represented as

$$
\begin{aligned}
i_{C} & =C \frac{\mathrm{d} u_{C}}{\mathrm{~d} t}, \\
& =C \frac{\mathrm{d} u_{L}}{\mathrm{~d} t} .
\end{aligned}
$$

Applying equation (4) to equation (3), the voltage $u_{L}$ can be expressed as

$$
u_{L}=u_{0}-\frac{L_{1}}{Z_{L}} \frac{\mathrm{d} u_{L}}{\mathrm{~d} t}-L_{1} C \frac{\mathrm{d}^{2} u_{L}}{\mathrm{~d} t^{2}}+L_{1} \frac{\mathrm{d} i_{S}}{\mathrm{~d} t}
$$

If the voltage referring instruction $u_{\text {ref }}$ and the system current $i_{S}$ are both defined as sinusoidal variables, 


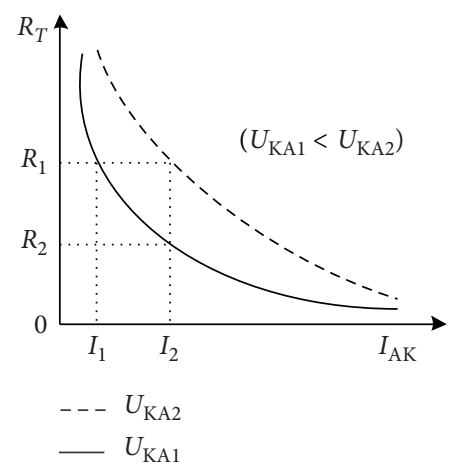

FIgURE 4: The equivalent relationship curves of resistance and current for thyristors.

$$
\left\{\begin{array}{l}
u_{\mathrm{ref}}=U_{m} \sin \omega t \\
i_{S}=I_{m} \sin (\omega t+\theta),
\end{array}\right.
$$

where $\omega$ is the angular frequency and $\theta$ is the phase angle. In addition, if $i_{S}$ decays to zero in a very short time $\Delta t$ and $u_{L}$ tracks the $u_{\text {ref }}$ with no deviation $\left(u_{L}=u_{\text {ref }}\right)$, then equation (5) can be converted as

$$
L_{1} \frac{I_{m} \sin (\omega t+\theta)}{\Delta t}=A \sin \omega t-B \cos \omega t
$$

where $A=\left(\omega^{2} L_{1} C+K_{U}-1\right) U_{m}, B=\omega U_{m} L_{1} / Z_{L}$.

Output voltage $u_{0}$ is replaced by $u^{\prime}=K_{U} U_{m} \sin \omega t$, which can be seen as an ideal linear proportional control model.

Then, the turn-off time of the conducting thyristor $\Delta t$ can be calculated as

$$
\Delta t=\frac{L_{1} I_{m} \sin (\omega t+\theta)}{U_{m}\left[\left(\omega^{2} L_{1} C+K_{U}-1\right) \sin \omega t-L_{1} \omega \cos \omega t / Z_{L}\right]} .
$$

Regardless of the unchangeable variables $\left(L_{1}, I_{m}, C, \omega, \theta\right.$, and $Z_{L}$ ), it is indicated that the turn-off time depends on three elements:

(1) Amplitude of the referring instruction, $U_{m}$

(2) Proportional control factor, $K_{U}$

(3) Occurring time of islanding transition, $t$

Anyway, the occurring time $t$ of an islanding event is unpredictable, but $U_{m}$ and $K_{U}$ can be calculated and then regulated by the controller. In equation (8), the decaying time of current $\Delta t$ is in an inverse proportion with $U_{m}$ and $K_{U}$, which means the bigger the value of $U_{m}$ and $K_{U}$, the faster the conducting thyristor will be turned off. Also, it is also corresponded with Figure 4 that the current $I_{\mathrm{AK}}$ has a greater equivalent resistance under $U_{\mathrm{KA} 2}$ when the value is greater than $U_{\mathrm{KA} 1}$.

The value of $\Delta u$ in equation (2) depends on the polarity of $i_{S}$, which can be recognized correctly only when the value of sampling is valid. As is shown in Figure 5, the hatched section is the linear region of sampling. For the positive part (vice versa), if the instantaneous value of the actual waveform is over the superior limit of the linear region sampling $\left(t_{2} \sim t_{3}\right)$, then the sampling value will be clamped in the maximum and

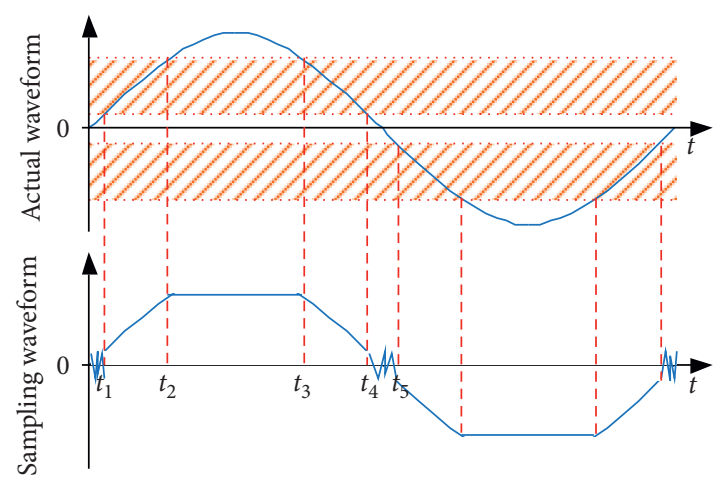

$\square \backslash$ Linear sampling region

FIGURE 5: Actual and resulting waveforms beyond the linear region of sampling.

the sampling waveform will be distorted. If the instantaneous value of the actual waveform is below the inferior limit of the linear sampling region $\left(0 \sim t_{1}\right.$ and $\left.t_{4} \sim t_{5}\right)$, then the sampling value will be overwhelmed by noise. Generally, different kinds of filter will be designed for the sampling data to obtain a smooth waveform. But, the phase delay of filters will also bring influence on the accuracy of polarity judgment.

For the studied system in this paper, when the instantaneous value of $i_{S}$ is near zero, the polarity is hard to be acquired accurately because of sampling noise. If the polarity of $i_{S}$ that the MG power controller detecting is contrary to actuality when islanding occurs, the voltage between the anode and the cathode of the thyristor is still positive and the thyristor will keep conducting. There are two consequences as follows.

On one hand, if the converter retains the current control strategy or shuts off its output until the conducting thyristor turns off, it will result in a longer shutdown time or an interruption of power supply for local loads even worse. On the other hand, if the converter changes the strategy to voltage control before the thyristor turning off completely, then it will connect parallelly with the public grid as a voltage source. In this case, it might cause a surge of current of the converter and a further deterioration for the voltage quality, which may trigger the local protection mechanism.

In order to get rid of the limitations of polarity detection mentioned above, a compulsory turn-off strategy based on fuzzy control of converter output voltage is proposed in this paper. This strategy is especially applicable when the polarity of the current flowing across the thyristor cannot be recognized accurately by the sampling circuit. In detail, an initial value of voltage reference instruction is given firstly to formulate a planned voltage and attempt to turn off the conducting thyristor. Then, the voltage reference and the control parameters will be adjusted dynamically according to the variation trend of the thyristors' current during the turn-off process.

\subsection{Islanding Transition Strategy with Fuzzy Control}

2.3.1. Foundation of Fuzzy Logic Control. The fuzzy logic controller relates the controller output and the inputs by 
using a list of inference rules. A basic structure of a typical fuzzy control block diagram is given in Figure 6. The inputs of the fuzzy controller consist of the deviation $e$ and the change rate of the deviation ec between the output $r$ and input $r^{*}$. The output of the fuzzy controller is carried out according to the fuzzy rules and is converted into variables that can be identified by the controlled system, which finally regulate the output.

2.3.2. Design of Fuzzy Control for Island Transition. According to the on-off characteristics of the thyristors, the decreasing speed of the current is related to the instantaneous value of itself before switching off and the reverse voltage between the anode and cathode. The smaller the instantaneous current value and the larger the reverse voltage are, the faster the current attenuation will be. As shown in Figure 3, the voltage $u_{a}$ is clamped by the utility. However, a fuzzy controller can be designed according to the basic characteristics of thyristors and the connecting relation of the MG system, with which the current $i_{S}$ can be regulated through $u_{L}$ indirectly and the referring instruction can be adjusted dynamically according to the instantaneous value of $i_{S}$ in order to realize the compulsory turnoff of the thyristors. In addition, the output voltage of the MG converter, which is working as a voltage source, is also affected by the closed-loop control parameters. Hence, as shown in Figure 7 , the inputs of the fuzzy controller are set as the deviation value and its rate of change between two adjacent sampling values of $i_{S}$, and the outputs of the fuzzy controller are the amount of changes for the amplitude of the instruction $u_{\text {ref }}$ and the voltage closed-loop control factor $K_{p}$.

The inputs of the fuzzy controller $e(k)$ and $e c(k)$ are obtained, respectively, as equations (9) and (10), of which the values are directly related to the instantaneous current $i_{S}$ and the sampling period $T$. To prevent an overcurrent between two adjacent sampling points in time, $T$ and $\Delta U$ should be restricted as equation (11), where $Z_{S}$ is the short-circuit impedance of the utility and $I_{P}$ is the trigger threshold of overcurrent protection for the MG system.

$$
\begin{aligned}
e(k) & =\left|i_{s}(k)\right|-\left|i_{s}(k-1)\right|, \\
e c(k) & =\frac{\mathrm{d} e(k)}{\mathrm{d} t} \\
& =\frac{e(k)-e(k-1)}{T}, \\
\frac{\Delta U}{T\left|Z_{S}\right|} & \leq I_{P} .
\end{aligned}
$$

Due to the actual range of the inputs and outputs variables, the basic level in the continuous domain and the quantitative level in discrete domains are shown in Table 1, respectively.

The fuzzy subset of $e, e c$, and $\Delta U$ are set as $\{\mathrm{NB}, \mathrm{NM}, \mathrm{NS}$, PS, PM, PB $\}$ and the subset of $\Delta K_{p}$ is set as $\{\mathrm{NB}, \mathrm{NM}, \mathrm{NS}$, ZE, PS, PM, PB . In addition, each input and output follows the triangle subjection function, which is shown in Figures 8(a) and 8(b).
2.3.3. Fuzzy Logic Rules. According to the fuzzy idea of compulsory turnoff and the principle of "if $A$ and $B$ and then $C^{\prime \prime}$, the fuzzy control rule is shown in Tables 2 and 3.

If the deviation as well as its rate of change between the absolute values of two adjacent sampling for $i_{S}$ is positive and increasing, it indicates that the voltage on the anode and the cathode of the thyristor is still positive as a conducting state. In this case, the polarity of $\Delta U$ should be changed. If the rate of change tends to decrease with positive deviation, the amplitude of $\Delta U$ should be increased properly. If the deviation change of $i_{s}$ is almost constant or increasing gradually, $\Delta K_{p}$ and $\Delta U$ should be both increased so that the divergent trend of $i_{s}$ can be slowed down. But, an improper value of $\Delta K_{p}$ will bring unexpected overshoot during the control process and affect the stability of the voltage output by the MG converter. So, $\Delta K_{p}$ should be selected properly.

If the deviation between the absolute values of two adjacent sampling for $i_{S}$ is negative, it indicates that the current $i_{s}$ has begun to move towards zero. When the changing rate of $i_{S}$ is large, it indicates that the A-K current is decaying very fast, then the parameter should be just kept as the former size. If the changing rate of $i_{S}$ decreases from a large value to a small value gradually, the amplitude of $\Delta U$ can be increased properly to accelerate the decay of $i_{s}$. In addition, the value of $\Delta K_{p}$ should increase properly if the deviation of $i_{S}$ keeps a small rate.

The referencing instruction of the voltage and close-loop controlling coefficient after regulating by the fuzzy control can be obtained as follows:

$$
\begin{gathered}
u_{\text {ref }}(k)=u_{\text {ref }}(k-1)+\Delta U(k), \\
K_{p}(k)=K_{p}(k-1)+\Delta K_{p}(k) .
\end{gathered}
$$

\section{Simulation and Results}

Simulated performances of the proposed strategy for compulsory islanding transition are evaluated in this section compared to the cases of natural turnoff and traditional forced turnoff with false polarity detecting. As discussed before, there are four possible combinations with different polarities of $i_{S}$ and $u_{S}$ when an islanding event occurs. To demonstrate the implementation process of the studied strategy, a specific time is selected, respectively, for instance in each case. In spite of different loads, it is universally applicable to any other time when the instantaneous values of $i_{S}$ and $u_{S}$ meet each condition of polarities under the four scenarios mentioned in equation (1).

Assume that, initially, the MG converter is operating in the grid-connected mode and each scenario takes the same initial value of $\Delta U$. Before the sag of the utility, there is simply a small deviation between the utility and the local loads voltage, which can also be seen as the sum of thyristor conduction voltage and line loss drop equivalently. Main parameters of the simulation model are in Table 4.

3.1. Natural Turnoff. Figure 9 shows the natural turn-off process of thyristors when there is voltage sag at an instant of $0.0601 \mathrm{~s}$. It can be discovered from Figure 9(a) that it takes 


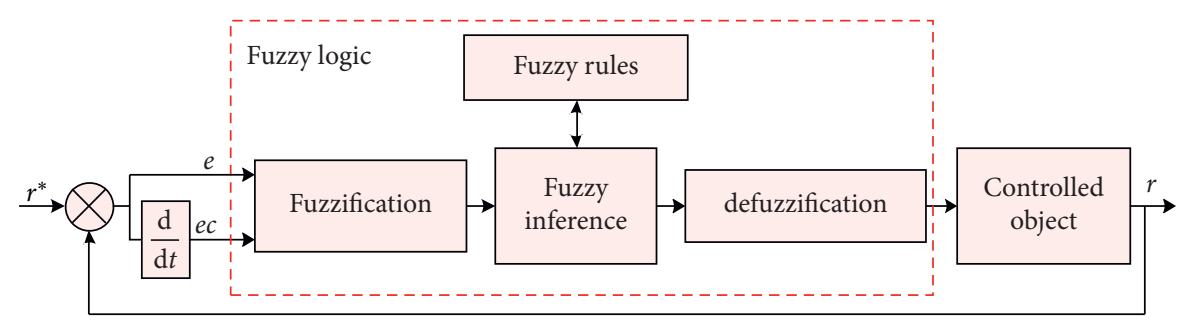

Figure 6: Block diagram of fuzzy control.

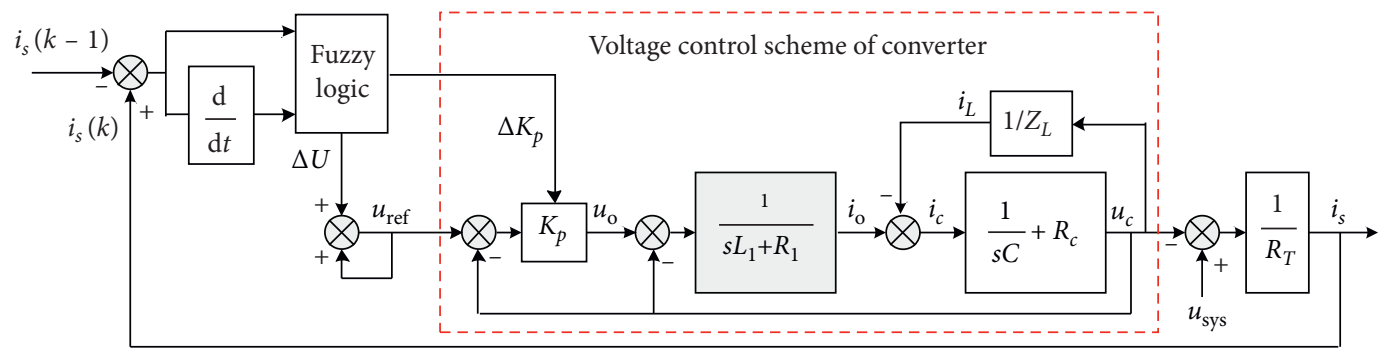

Figure 7: Control diagram of fuzzy control for islanding transition.

TABLE 1: The basic level and the quantitative level of variables in fuzzy control.

\begin{tabular}{lcr}
\hline Variables & Basic level & Quantitative level \\
\hline$e$ & $\{-60,60\}$ & $\{-3,-2,-1,1,2,3\}$ \\
$e c$ & $\{-200,200\}$ & $\{-3,-2,-1,1,2,3\}$ \\
$\Delta U$ & $\{-60,60\}$ & $\{-3,-2,-1,1,2,3\}$ \\
$\Delta K_{p}$ & $\{-3,3\}$ & $\{-3,-2,-1,0,1,2,3\}$ \\
\hline
\end{tabular}

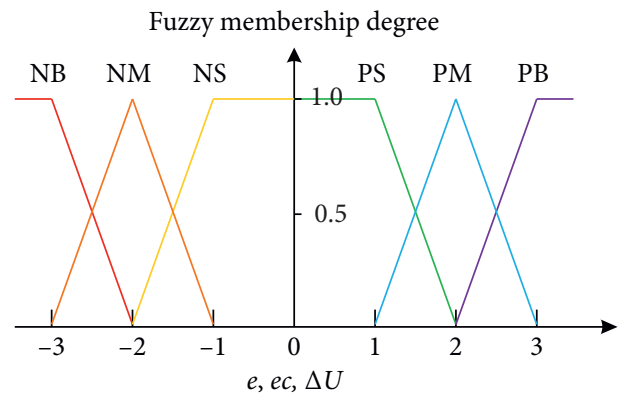

(a)

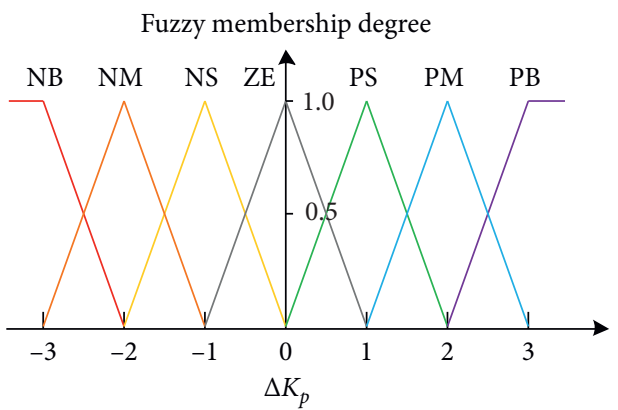

(b)

FIGURE 8: Membership functions of the input and output variables of the fuzzy controller: (a) Membership functions of $e, e c$, and $\Delta U$. (b) Membership functions of $\Delta K p$.

TABLE 2: Fuzzy control rule of $\Delta U$.

\begin{tabular}{|c|c|c|c|c|c|c|c|}
\hline & \multirow{2}{*}{$\Delta U$} & \multicolumn{6}{|c|}{$\mathrm{EC}$} \\
\hline & & $\mathrm{NB}$ & NM & NS & PS & $\mathrm{PM}$ & $\mathrm{PB}$ \\
\hline \multirow{6}{*}{$\mathrm{E}$} & $\mathrm{NB}$ & NM & NM & NB & $\mathrm{PB}$ & PM & $\mathrm{PM}$ \\
\hline & NM & NS & NS & NM & $\mathrm{PM}$ & PS & PS \\
\hline & NS & NS & NS & NS & PS & PS & PS \\
\hline & PS & NS & NS & NM & NM & NB & NB \\
\hline & PM & NS & NM & NM & NB & NB & NB \\
\hline & $\mathrm{PB}$ & NM & NM & NM & NB & NB & NB \\
\hline
\end{tabular}


TABLE 3: Fuzzy control rule of $\Delta K_{p}$.

\begin{tabular}{|c|c|c|c|c|c|c|c|}
\hline & \multirow{2}{*}{$\Delta K_{p}$} & \multicolumn{6}{|c|}{ EC } \\
\hline & & NB & $\mathrm{NM}$ & NS & PS & PM & $\mathrm{PB}$ \\
\hline \multirow{6}{*}{$\mathrm{E}$} & NB & $\mathrm{PB}$ & $\mathrm{PM}$ & PS & $\mathrm{ZE}$ & $\mathrm{ZE}$ & NB \\
\hline & $\mathrm{NM}$ & $\mathrm{PM}$ & PS & $\mathrm{ZE}$ & ZE & $\mathrm{ZE}$ & NS \\
\hline & NS & PS & $\mathrm{ZE}$ & $\mathrm{ZE}$ & $\mathrm{ZE}$ & NS & NM \\
\hline & PS & NS & $\mathrm{ZE}$ & $\mathrm{ZE}$ & $\mathrm{ZE}$ & $\mathrm{ZE}$ & PS \\
\hline & $\mathrm{PM}$ & $\mathrm{ZE}$ & PS & $\mathrm{PM}$ & $\mathrm{PM}$ & $\mathrm{PM}$ & $\mathrm{PB}$ \\
\hline & $\mathrm{PB}$ & PS & PS & PM & $\mathrm{PM}$ & $\mathrm{PB}$ & PB \\
\hline
\end{tabular}

TABLE 4: Main parameters of simulation.

\begin{tabular}{lc}
\hline Parameters & Initial values (unit) \\
\hline Phase voltage value of the utility, $U_{\text {sys }}$ & $220(\mathrm{~V})$ \\
Frequency of the utility voltage, $f$ & $50(\mathrm{~Hz})$ \\
Effective value of voltage sag, $U_{\text {sag }}$ & $60(\mathrm{~V})$ \\
Resistance of simulated load, $R_{L}$ & $2(\Omega)$ \\
Inductance of simulated load, $L$ & $4(\mathrm{mH})$ \\
Inductor of the LC filter, $L_{1}$ & $0.4(\mathrm{mH})$ \\
Capacitor of the LC filter, $C$ & $20(\mu \mathrm{F})$ \\
Sampling frequency, $f_{s}$ & $10(\mathrm{kHz})$ \\
Voltage closed-loop control factor, $K_{p}$ & 4 \\
Adjustment of voltage control factor, $\Delta K_{p}$ & 0 \\
Adjustment of voltage reference, $\Delta U$ & $20(\mathrm{~V})$ \\
\hline
\end{tabular}

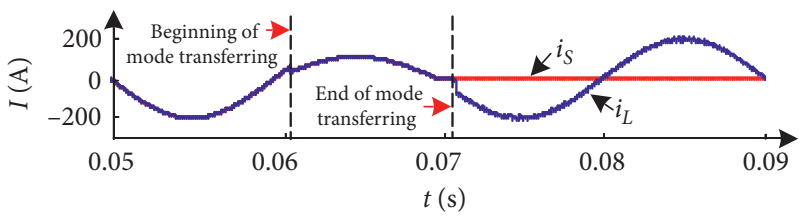

(a)

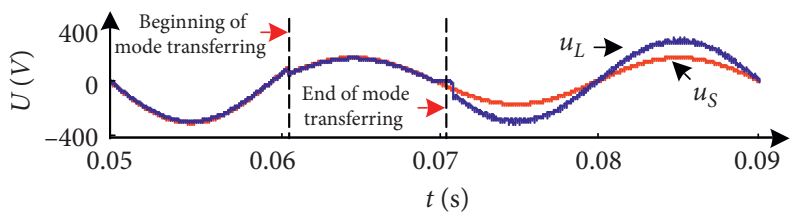

(b)

FIGURE 9: Simulation waveforms of natural turnoff for islanding transition: (a) simulation waveforms of the system and load current. (b) Simulation waveforms of the utility and load voltage.

almost half of the line cycle for the conducting thyristor to shut off completely. Then, the converter in the MG system works as a voltage source, which leads an interruption to continuity for the load in Figure 9(b).

\subsection{Compulsory Turnoff with False Polarity Detecting.} Figure 10 shows the unsuccessful disconnecting process when the recognition of current polarity is false. In Figure 10(a), the current $i_{S}$ is undergoing a surge during the turning-off process of the conducting thyristor in that case. Furthermore, as shown in Figure 10(b), the output voltage of the converter is seriously deteriorated because of the shortterm large current injection. After that, distortion is brought to the load current and the continuity and stability of the load operation are affected at the same time, which is consistent with the foregoing content in this paper.

\subsection{Successful Compulsory Disconnection}

3.3.1. Islanding Transition at the Moment When $i_{S}>0$ and $u_{S}>0$ (E.g., the Instant of $0.062 \mathrm{~s}$ ). If the polarity of the current $i_{S}$ is beyond the sampling linear region with positive polarity actually at the time of $0.062 \mathrm{~s}$ and the output voltage of the converter is equal to the referring instruction, then the condition of compulsory turnoff is satisfied as the initial value of $+20(\mathrm{~V})$ for $\Delta U$. So, the current $i_{S}$ decays to zero rapidly as is shown in Figure 11(a). Figure 11(b) shows that the value of $\Delta U$ has not changed for the stability of the MG system. When the thyristors have been turned off completely, the referring instruction will be switched to the state before the occurrence of islanding. Then, the converter will supply power for the local loads independently. It takes a very short time to obtain the whole process of islanding transfer and leads to no mutation or other abnormal scenarios no matter for the voltage or the current of local loads.

3.3.2. Islanding Transition at the Moment When $i_{S}>0$ and $u_{S}<0$ (E.g., the Instant of $0.0505 \mathrm{~s}$ ). If the islanding mode transfers at the time of $0.0505 \mathrm{~s}$, the initial value of $+20(\mathrm{~V})$ for $\Delta U$ will lead the thyristor to continue to conduct. Then, the current $i_{\mathrm{S}}$ tends to increase, as is shown in Figure 12(a). When the MG power controller detects that the thyristor current does not decay, the fuzzy controller adjusts the polarity of $\Delta U$, and the converter reference instruction responds to the change of polarity quickly. Figure 12(b) shows 


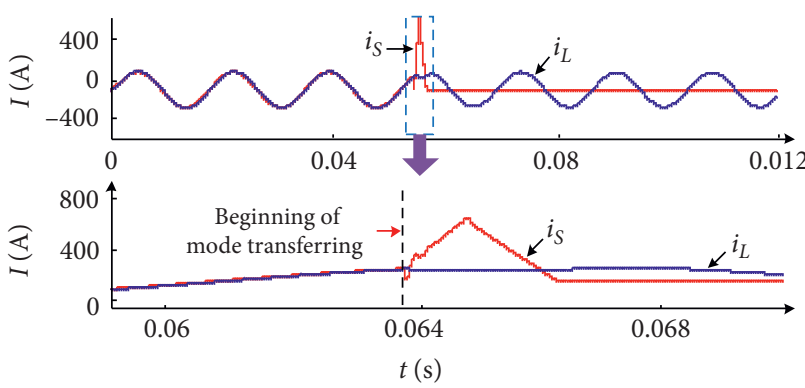

(a)

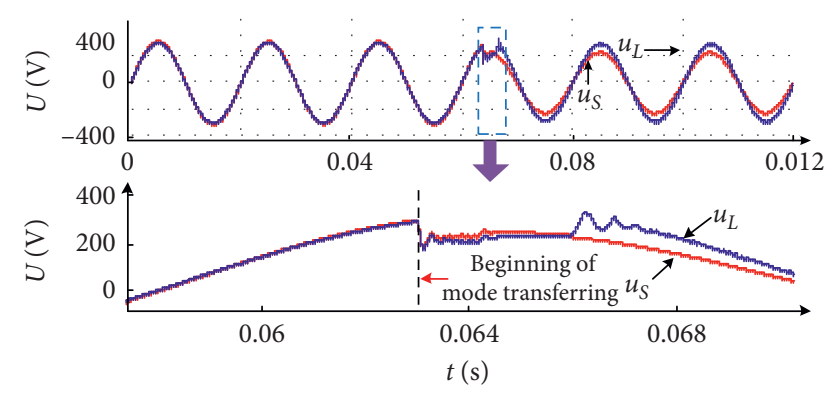

(b)

FIGURE 10: Simulation waveforms of compulsory turnoff with false polarity detecting: (a) simulation waveforms of the system and load current. (b) Simulation waveforms of the utility and load voltage.

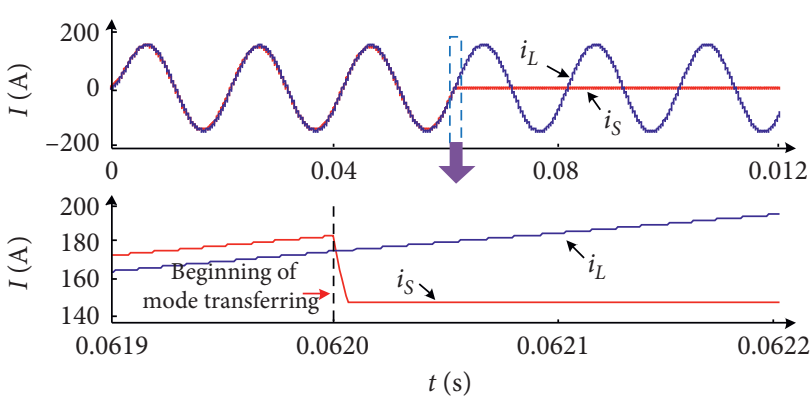

(a)

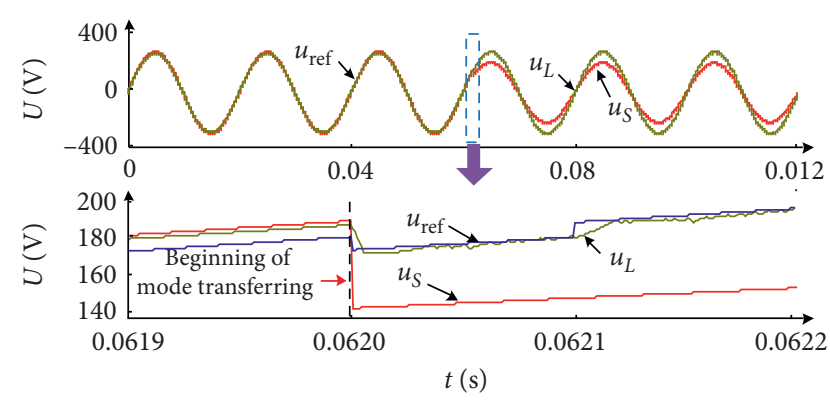

(b)

FIGURE 11: Simulation waveforms of islanding transition at time $0.062 \mathrm{~s}$ : (a) simulation waveforms of the system and load current. (b) Simulation waveforms of the utility, reference, and load voltage.

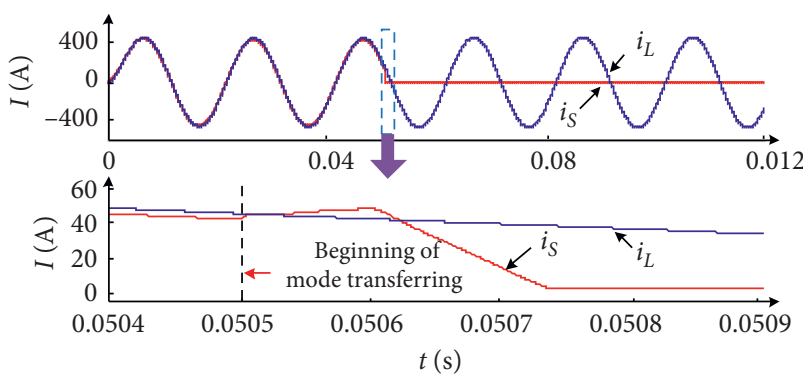

(a)

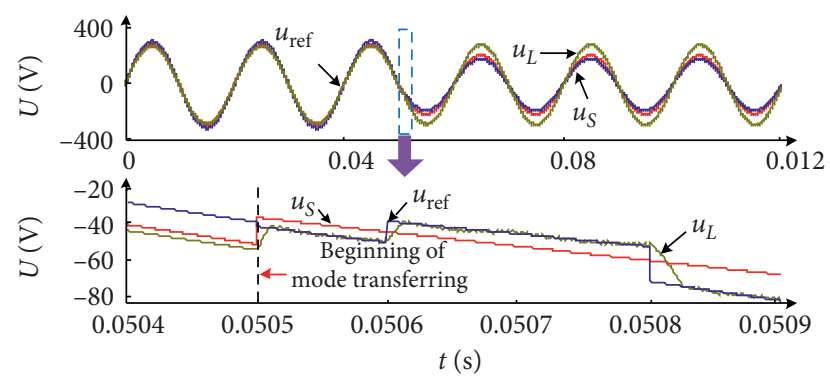

(b)

FIGURE 12: Simulation waveforms of islanding transition at time $0.0505 \mathrm{~s}$ : (a) simulation waveforms of the system and load current. (b) Simulation waveforms of the utility, reference, and load voltage.

that the current $i_{S}$ begins to decay as the backpressure between the anode and cathode rapidly appears after the output voltage of the converter tracking the reference instruction. At the time of $0.0508 \mathrm{~s}$, the conducting thyristor can be considered turned off completely. Then, the converter readjusts the reference instruction to provide standard voltage to local loads.

3.3.3. Islanding Transition at the Moment When $i_{S}<0$ and $u_{S}>0$ (E.g., the Instant of $0.0615 \mathrm{~s}$ ). Figure 13 shows the islanding mode transfer occurs when the instantaneous value of the current $i_{S}$ is negative and the utility voltage is positive. Because the initial value of $\Delta U$ is $+20(\mathrm{~V})$ and the initial situation is considered to be $u_{L}>u_{S}$, the condition of compulsory turnoff cannot be met and the current $i_{S}$ experiences a trend of gradual increase in Figure 13(a). After detecting the abnormal increasing current, the fuzzy controller adds the opposite polarity of $\Delta U$ to the voltage referring instruction to obtain the reserve voltage in a very short time.

3.3.4. Islanding Transition at the Moment When $i_{S}<0$ and $u_{S}<0$ (E.g., the Instant of $0.0059 \mathrm{~s}$ ). Similar to the curves in Figure 11, the initial value of $\Delta U$ makes voltage across the conducting thyristor negative at the time of $0.059 \mathrm{~s}$, which can ensure the compulsory turn-off process. In Figure 14(a), 


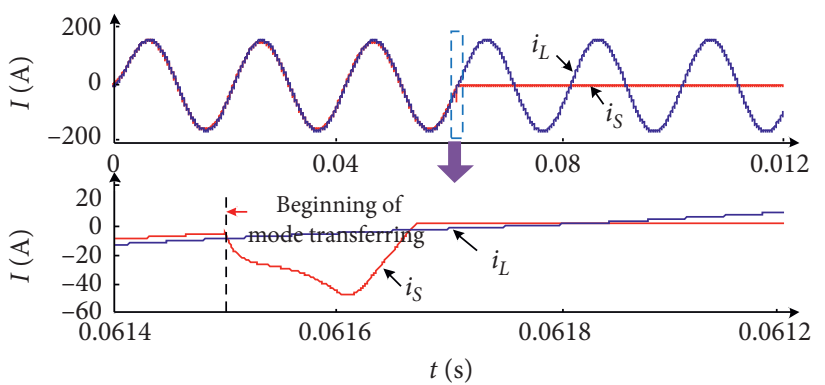

(a)

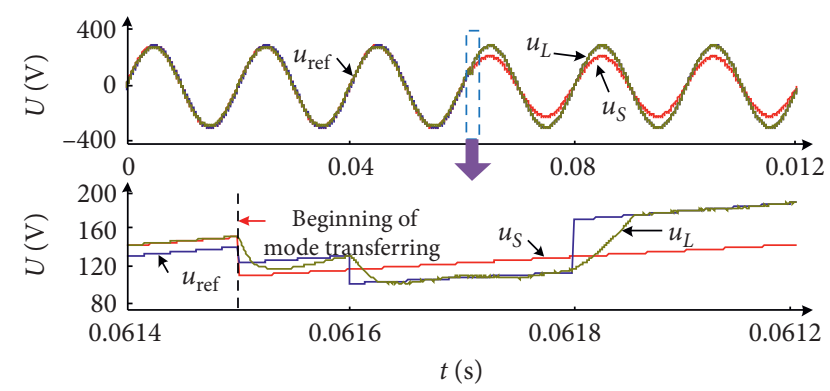

(b)

Figure 13: Simulation waveforms of islanding transition at time $0.0615 \mathrm{~s}$ : (a) simulation waveforms of the system and load current. (b) Simulation waveforms of the utility, reference, and load voltage.

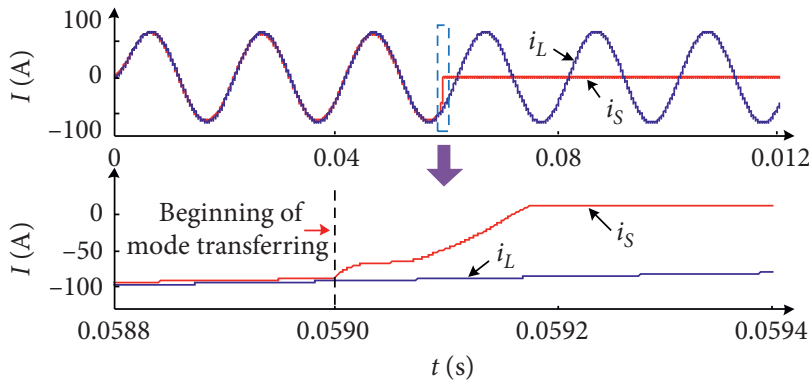

(a)

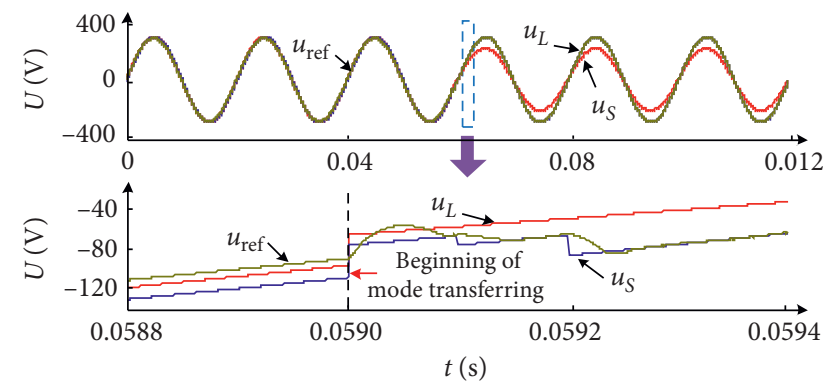

(b)

FigURE 14: Simulation waveforms of islanding transition at time $0.059 \mathrm{~s}$ : (a) simulation waveforms of the system and load current. (b) Simulation waveforms of the utility, reference, and load voltage.

the current $i_{S}$ does not increase to the reverse direction but falls rapidly. Meanwhile, it is shown in Figure 14(b) that the original parameters of voltage control closed-loop fail to make the output voltage track referring instruction well and the changing rate of the current $i_{S}$ is declined at the beginning of the compulsory turn-off process. So, the proportional coefficient $K_{p}$ is regulated by the designed fuzzy controller in order to improve the dynamic response speed of the voltage control loop.

\section{Practical Experiment and Results}

Practical experiment is conducted to prove the effectiveness of the disconnecting strategy proposed in this paper. In actual MG systems, the kinds of sensitive loads are diverse and the voltage-current curves vary as well. Without loss of generality, a common resistance type and a nonlinear singlephase rectifier bridge are selected as the experimental load with low current level, so the time of disconnection can be unspecified in practical experiment to verify the applicability of the studied strategy. Parameters of the controller are the same as in Table 4.

Figure 15 shows the load current with natural turnoff of thyristors when the MG system disconnects from the utility. As it can be seen, the load current is discontinuous and the stand-alone mode of the MG system begins until load current reaches the first zero crossing. It takes almost $1 / 4$ fundamental cycle. For sensitive loads, this kind of discontinuity might be intolerable.

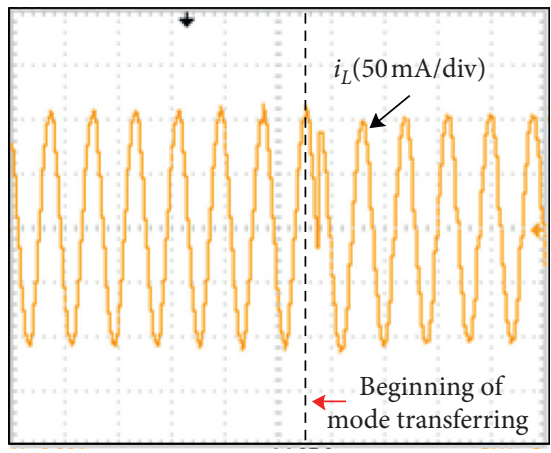

FIGURE 15: Waveform of load current with natural turnoff for thyristors.

Figure 16 shows the system current $i_{S}$ and the load current $i_{L}$ during the transferring from the utility-connected mode to stand-alone mode when the recognition of polarity is incorrect. It shows that the thyristor is still conducting when the recognition of polarity for the current $i_{S}$ is incorrect and undergoes an inrush. Meanwhile, the load current is oscillating periodically, which will affect the stable operation of the load.

With the strategy proposed in this paper, experimental results based on fuzzy logic indicate the correctness and validity in Figures 17 and 18 with linear load and nonlinear load, respectively. It shows that the transient progress of islanding barely bring any impact on the continuity and 


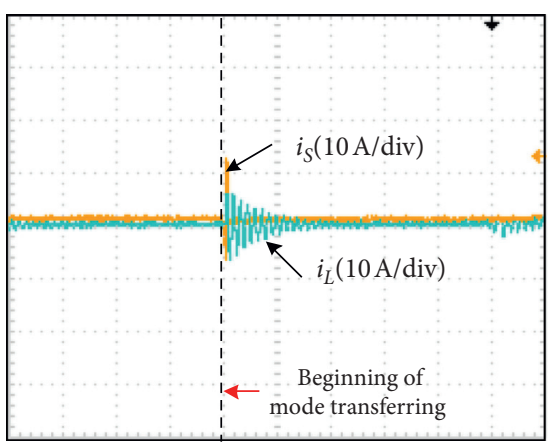

FIGURE 16: Waveforms of the system and load current with compulsory turnoff when the recognition of polarity is incorrect.

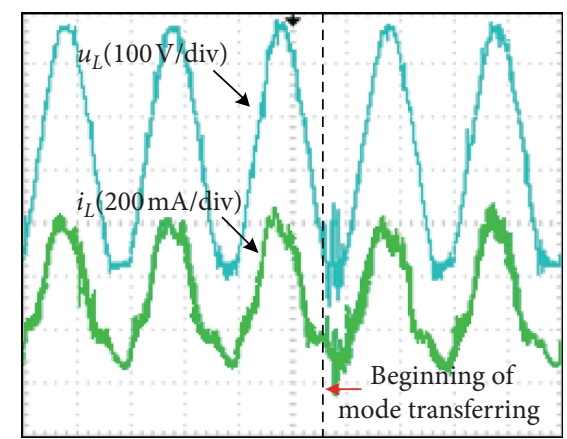

Figure 17: Waveforms of voltage and current with compulsory turnoff for linear load.

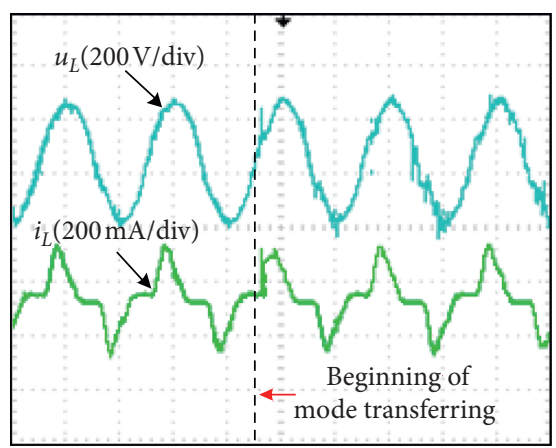

FIGURE 18: Waveforms of voltage and current with compulsory turnoff for nonlinear load.

stability of load without detecting the polarity of voltage or current, which can be accepted for almost all of the existing sensitive loads.

\section{Conclusions}

For the microgrid system, the mode transition, especially when it disconnects from the utility, must be transient, seamless, and reliable. If the current exceeds the linear sampling region of the system, it is difficult to ensure the rapidity and stability of mode transition.

In this paper, it proposes a compulsory turn-off strategy for the thyristors when the MG system disconnects from the utility. Rather than detecting the voltage or current polarity, the proposed strategy can realize fast turning off for the thyristors at any time by adjusting the converter reference instruction and closedloop control parameters dynamically with fuzzy logic. Then, the disconnection process will not be affected by the software or hardware of the MG system and will be more applicable and efficient. However, a more sophisticated strategy can be extended from future studies, and there are still problems, which need to be further researched at the same time.

\section{Nomenclature}

MG: $\quad$ Microgrid

DG: Distributed generating

BS: $\quad$ Battery storing

SSR: $\quad$ Solid-state relay

PI: $\quad$ Proportional integral

SVPWM: Space vector pulse width modulation

NB: $\quad$ Negative big

NM: $\quad$ Negative middle

NS: $\quad$ Negative small

PS: $\quad$ Positive small

PM: $\quad$ Positive middle

PB: $\quad$ Positive big

$u_{S}, i_{S}: \quad$ Voltage and current of the utility $(\mathrm{V}, \mathrm{A})$

$I_{m}: \quad$ Amplitude of $i_{S}(\mathrm{~A})$

$Z_{S}: \quad$ Equivalent impedance of the utility $(\Omega)$

$u_{L}, i_{L}: \quad$ Voltage and current of local loads $(\mathrm{V}, \mathrm{A})$

$Z_{L}: \quad$ Equivalent impedance of local loads $(\Omega)$

$u_{0}, i_{0}$ : Output voltage and output current of the converter $(\mathrm{V}, \mathrm{A})$

$L_{1}, R_{1}: \quad$ Inductor and parasitic resistance of the LC filter

$C, R_{\mathrm{c}}: \quad$ Capacitor and parasitic resistance of the LC filter $(\mathrm{F}, \Omega)$

$i d_{\text {ref }}, i q_{\text {ref: }}$ Current reference in the $d$-axis and $q$-axis (A)

$i_{0} d, i_{0} q$ : Component of $i_{0}$ in the $d$-axis and $q$-axis (A)

$u_{\text {ref: }} \quad$ Voltage reference of the converter $(\mathrm{V})$

$U_{m}: \quad$ Amplitude of voltage reference (V)

$i^{*}: \quad$ Current reference of the voltage control loop (A)

$i_{g}: \quad$ Grid-connected current of the converter (A)

$u_{a}$ : $\quad$ Voltage near the side of the utility

$u b$ : Voltage near the side of the MG converter

$R_{T}$ : $\quad$ Equivalent resistance of the thyristor when shutting off $(\Omega)$

$U_{\mathrm{AK}}, I_{\mathrm{AK}}$ : Anode-cathode voltage and current of the thyristor $(\mathrm{V}, \mathrm{A})$

$U_{\mathrm{KA}}: \quad$ Cathode-anode voltage of the thyristor $(\mathrm{V})$

$K_{U}: \quad$ Equivalently proportional coefficient

$e, e c$ : Inputs of the fuzzy controller

$\Delta U, \Delta K_{p}$ : Outputs of the fuzzy controller

$K_{p}$ : $\quad$ Proportional coefficient of the voltage loop.

\section{Data Availability}

The data used to support the findings of this study are included within the article. 


\section{Conflicts of Interest}

The authors declare that there are no conflicts of interest regarding the publication of this paper.

\section{References}

[1] E. Unamuno and J. A. Barrena, "Hybrid AC/DC microgridspart I: review and classification of topologies," Renewable and Sustainable Energy Reviews, vol. 52, pp. 1251-1259, 2015.

[2] A. Cagnano, E. D. Tuglie, and P. Mancarella, "Microgrids: overview and guidelines for practical implementations and operation," Applied Energy, vol. 258, Article ID 114039, 2020.

[3] P. Li, X. Guan, J. Wu, and D. Wang, "An integrated energy exchange scheduling and pricing strategy for multi-microgrid system," in Proceedings of the IEEE Region 10 Conference, pp. 1-5, IEEE, Xi'an, China, October 2013.

[4] D. S. Ramos, T. E. D. Carpio-Huayllas, and R. L. VasquezArnez, "Load shedding application within a microgrid to assure its dynamic performance during its transition to the islanded mode of operation," Energy and Power Engineering, vol. 5, no. 7, pp. 437-445, 2013.

[5] I. Serban, "A control strategy for microgrids: seamless transfer based on a leading inverter with supercapacitor energy storage system," Applied Energy, vol. 221, pp. 490-507, 2018.

[6] S. Huang, W. Pei, and L. Kong, "Control algorithm research on seamless transfer for PV system during intentional islanding," in Proceedings of the ISES Solar World Congress 2007 III, pp. 1637-1641, Springer, Beijing, China, September 2008.

[7] R. Tirumala, N. Mohan, C. Henze, and I. O. E. A. Engineer, "Seamless transfer of grid-connected PWM inverters between utility-interactive and stand-alone modes," in Proceedings of the Applied Power Electronics Conference and Exposition Seventeenth Annual IEEE, vol. 2, pp. 1081-1086, IEEE, Dallas, TX, USA, March 2002.

[8] B. Singh, G. Pathak, and B. K. Panigrahi, "Seamless transfer of renewable-based microgrid between utility grid and diesel generator," IEEE Transactions on Power Electronics, vol. 33, no. 10, pp. 8427-8437, 2018.

[9] C. Chang and Y. Chang, "Energy storage systems for seamless mode transfer in microgrid," in Proceedings of the 2011 IEEE International Conference on Power Electronics and Drive Systems, pp. 799-802, Singapore, December 2011.

[10] L. Jia, Y. Zhu, S. Du, and Y. Wang, "Analysis of the transition between multiple operational modes for hybrid AC/DC microgrids," CSEE Journal of Power and Energy Systems, vol. 4, no. 1, pp. 49-57, 2018.

[11] I. Ahmed, L. Sun, and X. Chen, "A novel control scheme for microgrid inverters seamless transferring between grid-connected and islanding mode," in Proceedings of the 2017 (China) International Electrical and Energy Conference, pp. 75-80, IEEE, Beijing, China, October 2017.

[12] M. Abadi and S. M. Sadeghzadeh, "A control approach with seamless transition capability for a single-phase inverter operating in a microgrid," International Journal of Electrical Power \& Energy Systems, vol. 111, pp. 475-485, 2019.

[13] G. Lou, W. Gu, J. Zhu, P. Li, and X. Zhang, "A novel control strategy for the seamless transfer of microgrids based on disturbance observer," International Journal of Electrical Power \& Energy Systems, vol. 118, Article ID 105804, 2020.

[14] A. H. Etemadi and R. Iravani, "Supplementary mechanisms for smooth transition between control modes in a microgrid," Electric Power Systems Research, vol. 142, pp. 249-257, 2017.
[15] W. Issa, A. E. Khateb, N. Anani, and M. Abusara, "Smooth mode transfer in AC microgrids during unintentional islanding," Energy Procedia, vol. 134, pp. 12-20, 2017.

[16] W. Zhang, H. Liu, W. Wang, and P.C. Loh, "Seamless transfer scheme for parallel PV inverter system," IET Power Electronics, vol. 13, no. 5, Article ID 101049, 2020.

[17] J. Hmad, A. Houari, H. Trabelsi, and M. Machmoum, "Fuzzy logic approach for smooth transition between grid-connected and stand-alone modes of three-phase DG-inverter," Electric Power Systems Research, vol. 175, Article ID 105892, 2019.

[18] M. Ganjian-Aboukheili, M. Shahabi, Q. Shafiee, and J. M. Guerrero, "Seamless transition of microgrids operation from grid-connected to islanded mode," IEEE Transactions on Smart Grid, vol. 11, no. 3, pp. 2106-2114, 2020.

[19] H. Ameli, E. Abbasi, M. T. Ameli, and G. Strbac, "A fuzzylogic-based control methodology for secure operation of a microgrid in interconnected and isolated modes," International Transactions on Electrical Energy Systems, vol. 27, no. 11, Article ID e2389, 2017.

[20] H. Lhachimi, Y. Sayouti, and Y. E. Kouari, "Control of a flexible microgrid during both modes of operations with presence of nonlinear loads," Journal of the Franklin Institute, vol. 357, no. 11, pp. 6498-6538, 2020.

[21] S. Choudhury, T. P. Dash, P. Bhowmik, and P. K. Rout, “A novel control approach based on hybrid fuzzy logic and seeker optimization for optimal energy management between microsources and supercapacitor in an islanded microgrid," Journal of King Saud University-Engineering Sciences, vol. 32, no. 1, pp. 27-41, 2020.

[22] J. P. Roselyn, A. Ravi, D. Devaraj, R. Venkatesan, M. Sadees, and K. Vijayakumar, "Intelligent coordinated control for improved voltage and frequency regulation with smooth switchover operation in LV microgrid," Sustainable Energy, Grids and Networks, vol. 22, Article ID 100356, 2020.

[23] J. Xiang, F. Ji, H. Nian, J. Zhang, and H. Deng, "Seamless transfer of single-phase utility interactive inverters with a synchronized output regulation strategy," Journal of Power Electronics: A Publications of The Korean Institute Of Power Electronics, vol. 16, no. 5, pp. 1821-1832, 2016.

[24] X. Liao, Z. Guo, and D. Sha, "Voltage magnitude and frequency control of three-phase voltage source inverter for seamless transfer," IET Power Electronics, vol. 7, no. 1, pp. 200-208, 2014.

[25] C. Sun, G. Joos, S. Q. Ali et al., "Design and real-time implementation of a centralized microgrid control system with rule-based dispatch and seamless transition function," IEEE Transactions on Industry Applications, vol. 56, no. 3, pp. 3168-3177, 2020.

[26] S. Lee, K. Sung, T. Lee, and W. Lee, "UPS/APF power conversion equipment with a seamless mode transfer," in Proceedings of the IEEE 8th International Conference on Power Electronics, pp. 2769-2776, IEEE, Jeju, South Korea, June 2011.

[27] B. Kroposki, C. Pink, J. Lynch et al., "Development of a highspeed static switch for distributed energy and microgrid applications," in Proceedings of the 2007 Power Conversion Conference, pp. 1418-1423, IEEE, Nogaya, Japan, April 2007.

[28] G. F. Reed, M. Takeda, and I. Iyoda, "Improved power quality solutions using advanced solid-state switching and static compensation technologies," in Proceedings of the IEEE Power Engineering Society 1999 Winter Meeting, vol. 2, pp. 11321137, New York, NY, USA, February 1999.

[29] C. Chen, Y. Wang, J. Lai, Y. Lee, and D. Martin, "Design of parallel inverters for smooth mode transfer microgrid 
applications," IEEE Transactions on Power Electronics, vol. 25, no. 1, pp. 6-15, 2010.

[30] W. U. Tareen and S. Mekhilef, "Transformer-less 3P3W SAPF (three-phase three-wire shunt active power filter) with lineinteractive UPS (uninterruptible power supply) and battery energy storage stage," Energy, vol. 109, pp. 525-536, 2016.

[31] K.-R. Kim, C.-Y. Oh, T.-J. Kim, J.-P. Lee, and H.-J. Kim, "Implementation of a smart power conditioning system for energy storage system with a novel seamless transfer strategy," Energies, vol. 11, no. 5, p. 1108, 2018.

[32] K.-Y. Lo and Y.-M. Chen, "Design of a seamless grid-connected inverter for microgrid applications," IEEE Transactions on Smart Grid, vol. 11, no. 1, pp. 194-202, 2020.

[33] Y. Chen, Z. Wang, X. Zhou et al., "Seamless transfer control strategy for three-phase inverter in microgrid," in Proceedings of the IEEE 8th International Power Electronics and Motion Control Conference, pp. 1759-1763, IEEE, Hefei, China, May 2016.

[34] B. Tian, C. Mao, D. Wang et al., "Control strategy of gridconnected/grid-disconnected seamless transfer of energy storage converter in microgrid," Proceedings of the CSUEPSA, vol. 26, no. 11, pp. 1-27, 2014.

[35] L. Hou, L. Xu, Y. Wei et al., "Passive network optimization design of seamless switching of controllable switches in microgrid," Automation of Electric Power System, vol. 42, no. 7, pp. 171-177, 2018.

[36] G. Shen, D. Xu, and D. Xi, "Novel seamless transfer strategies for fuel cell inverters from grid-tied mode to off-grid mode," in Proceedings of the IEEE Applied Power Electronics Conference \& Exposition, vol. 1, pp. 109-113, IEEE, Austin, TX, USA, March 2005.

[37] N. W. A. Lidula and A. D. Rajapakse, "Behavior of a microgrid during the transision from grid connected to islanded mode," in Proceedings of the 2nd Annual International Conference on Sustainable Energy and Environmental Sciences, pp. 28-33, Singapore, February 2013. 